\title{
Autoeficácia psicossocial em jovens com Diabetes Mellitus e sua influência no autocuidado
}

\author{
Psychosocial self-efficacy in young people with diabetes mellitus and its influence on self- \\ care
}

Evelin Matilde Arcain Nass' ${ }^{1}$, Sonia Silva Marcon ${ }^{1}$, Elen Ferraz Teston ${ }^{2}$, Maria do Carmo Fernandez Lourenço Haddad $^{3}$, Pamela dos Reis ${ }^{1}$, Iven Giovanna Trindade Lino ${ }^{1}$

\begin{abstract}
Objetivo: identificar a autoeficácia psicossocial em jovens com Diabetes Mellitus tipo 1 e associação com variáveis sociodemográficas, perfil de saúde e busca por atendimento de saúde. Métodos: estudo transversal realizado com 35 jovens, mediante entrevista audiogravada. Usou-se dois instrumentos: a) roteiro de caracterização sociodemográfica, perfil de saúde e busca por atendimento em saúde; b) Escala de Autoeficácia em DiabetesVersão Curta. Na análise, utilizou-se estatística descritiva e inferencial - teste Qui-quadrado e Exato de Fisher. Resultados: a totalidade dos jovens apresentou índices satisfatórios de autoeficácia (moderada e alta). Embora sem significância estatística, observou-se a proporção ligeiramente maior de autoeficácia alta em jovens do sexo masculino, maiores de 18 anos, da cor branca, que não trabalham, com plano de saúde, acima do peso, com mais de oito anos de diagnóstico e que não sofreram hospitalização. Conclusão: os jovens apresentam autoeficácia satisfatória, não sendo observada associação significativa com variáveis sociodemográficas e clínicas.
\end{abstract}

Descritores: Enfermagem; Diabetes Mellitus; Autoeficácia; Autocuidado.

Objective: to identify psychosocial self-efficacy in young people with Type 1 diabetes mellitus and its association with sociodemographic variables, health profile, and search for health care. Methods: cross-sectional study conducted with 35 young people through audio-recorded interviews. Two instruments were used: a) script for characterization of sociodemographic data, health profile, and search for health care; b) Diabetes Management Self-Efficacy Scale - Short Version. In the analysis, we used descriptive and inferential statistics - Chi-square test and Fisher's Exact test. Results: all young people had satisfactory (moderate and high) self-efficacy levels. Although not statistically significant, there was a slightly higher proportion of high self-efficacy in young males, people over 18 years old, white skinned, unemployed, with overweight, with health insurance, with more than eight years of diagnosis, and not hospitalized. Conclusion: young people present satisfactory self-efficacy, without significant association with sociodemographic and clinical variables.

Descriptors: Nursing; Diabetes Mellitus; Self Efficacy; Self Care.

\footnotetext{
${ }^{1}$ Universidade Estadual de Maringá. Maringá, PR, Brasil.

${ }^{2}$ Universidade Federal do Mato Grosso do Sul. Campo Grande, MS, Brasil.

${ }^{3}$ Universidade Estadual de Londrina. Londrina, PR, Brasil. 


\section{Introdução}

O Diabetes Mellitus tipo 1 (DM1), anteriormente conhecido como Diabetes juvenil, compreende10,0\% do total de casos de Diabetes. Essa condição crônica, caracterizada pela deficiência absoluta de insulina, e suas causas estão relacionadas ao processo autoimune, que é desencadeado por uma interação de fatores genéticos e ambientais. Ele pode ocorrer em qualquer idade, mas em geral é diagnosticado antes dos 20 anos. Suas complicações podem acarretar invalidez precoce e diminuição na qualidade de vida, além de prejuízos econômicos causados pelo alto custo do tratamento e das frequentes hospitalizações ${ }^{(1)}$.

A infância e a adolescência são fases em que ocorre intenso desenvolvimento físico e psicológico que desencadeiam importantes transformações biopsicossociais. Sendo assim, a presença de uma condição crônica como o DM1 exige que o adolescente, além de lidar com as demandas próprias dessa etapa da vida, também atenda aquelas oriundas da doença e do tratamento, o que pode acarretar desordens emocionais que, se não devidamente trabalhadas, poderão influenciar negativamente sua qualidade de vida ${ }^{(2)}$.

A convivência com o DM1 exige do jovem adequação em seu padrão de vida, autosuperação, aceitação e resignação. Entretanto, a conscientização dessa necessidade é alcançada somente a partir do momento que a maturidade se desenvolve, além de considerar a fase em que se encontra a doença e o seu contexto. Assim, aos poucos, as responsabilidades anteriormente atribuídas aos responsáveis são progressivamente assumidas pelo jovem, o qual procura se adaptar ao novo estilo de $\operatorname{vida}^{(3)}$. Nesse contexto, é importante o estímulo às práticas de autocuidado, atentando-se para a necessidade de compreensão das condições do paciente e investigação dos fatores que interferem em seu tratamento.

Além da idade, vários outros fatores podem influenciar o indivíduo no enfrentamento do DM1, tais como: sexo, raça, nível de escolaridade, situação socioeconômica, familiar, ocupacional e a autoeficá- cia psicossocial ${ }^{(4)}$. Dentre estes fatores, a autoeficácia psicossocial vem se destacando como um importante comportamento de autocuidado e de controle glicêmico nos jovens com DM1 $1^{(5)}$.

A autoeficácia psicossocial refere-se à capacidade que o indivíduo apresenta para enfrentar as adversidades inerentes a uma determinada situação. Quando esta é moderada ou alta, pode associar-se a autoestima elevada, melhor qualidade de vida, de acompanhamento da doença e diminuição das barreiras na adesão ao tratamento em jovens com DM1 $1^{(5)}$. Assim, considerando a complexidade no manejo do DM1 e os obstáculos da doença crônica, em especial na juventude, torna-se relevante conhecer a autoeficácia dos jovens acometidos, pois esta constitui indicador da qualidade do autogerenciamento da doença. Este conhecimento poderá subsidiar a implementação de uma assistência em saúde mais adequada a esta população. Deste modo, definiu-se como objetivo identificar a autoeficácia psicossocial em jovens com Diabetes Mellitus tipo 1 e associação com variáveis sociodemográficas, perfil de saúde e busca por atendimento de saúde.

\section{Métodos}

Estudo transversal, parte de outro maior realizado com jovens com DM1. O cenário do estudo foi a cidade de Maringá- PR, que à época possuía população estimada de 406.693 habitantes, sendo 116.415 jovens ${ }^{(6)}$. Os jovens com DM1 foram localizados a partir de uma lista de pacientes com DM fornecida pela Secretaria Municipal de Saúde, contendo nome completo, idade e sexo. Nesta lista, identificou-se 99 pessoas na faixa etária de 15 a 29 anos, e em consulta ao Prontuário Eletrônico, obteve-se, para contato, seus endereços e telefones.

Foram convidados a participar do estudo mediante ligação telefônica, ocasião em que se verificou o atendimento aos seguintes critérios de inclusão: confirmação do diagnóstico de DM estabelecido a qualquer tempo, residir no município e ter conta no 
Facebook ${ }^{\circledR}$. 0 único critério de exclusão estabelecido foi não ser localizado em pelo menos cinco tentativas em dias e horários diversos. Deste modo, 35 jovens participaram do estudo, visto que oito referiram não ter DM, 20 se recusaram a participar, 18 residiam em outro município, 12 não foram localizados e seis não tinham conta no Facebook $^{\circledR}$, requisito necessário para a segunda etapa do estudo que previa uma intervenção utilizando rede social. (Não cabe apresentar detalhes da outra etapa nesta comunicação)

Os dados desta comunicação foram coletados no período de fevereiro a maio de 2017 mediante entrevista presencial e audiogravada, com duração média de 25 minutos. As entrevistas foram realizadas nos locais de preferência dos participantes, sendo $25 \mathrm{em}$ seus domicílios, sete na faculdade e três no local de trabalho. Durante as entrevistas, utilizou-se dois instrumentos: a) roteiro para caracterização de variáveis sociodemográficas, perfil de saúde, estado nutricional e busca por atendimento de saúde; b) Escala de Autoeficácia em Diabetes-Versão $\operatorname{Curta}^{(7)}$, que mede a autoeficácia psicossocial para o gerenciamento do autocuidado em DM. 0 estado nutricional foi definido de acordo com o índice de massa corporal, calculado a partir da idade e das informações autorreferidas de peso e altura, sendo classificado em adequado e excesso de peso ${ }^{(8)}$.

A Escala é constituída por oito questões relacionadas à mudança de comportamento, elaboração de um plano de cuidados, superação de barreiras, solicitação de apoio, cuidar de si, gestão emocional, motivações pessoais, e a escolha por decisões adequadas sobre o cuidado da diabetes ${ }^{(7)}$. As respostas são registradas em uma escala do tipo Likert de cinco pontos que variam de "estou muito de acordo" (cinco pontos) a "não estou de acordo de jeito nenhum" (um ponto). Os valores numéricos para o conjunto de itens são somados e divididos por oito. É considerada baixa eficácia uma pontuação entre um e 2,3; moderada, entre 2,4 e 3,7 e alta entre 3,8 e 5,0 $0^{(7)}$. No presente estudo, considerou-se autoeficácia satisfatória as classificadas como moderada e alta. 0 instrumento foi adaptado para o público jovem com alteração apenas do pronome de tratamento, substituindo "Senhor (a)" por "Você".

Os dados foram inseridos em um banco no software Microsoft Excel $^{\circledR}$ e, em seguida, transferidos e analisados no Statistical Package for the Social Sciences (versão 20.0 for Windows), utilizando a estatística descritiva e inferencial, com uso de média e desvio padrão. 0 teste Qui-quadrado e Exato de Fisher foram utilizados para verificar a associação entre a variável dependente (autoeficácia) e as demais variáveis do estudo (comportamentos adotados em relação ao tratamento, dados sociodemográficos, perfil de saúde e busca por atendimento), sendo considerado significante quando $\mathrm{p} \leq 0,05$.

No desenvolvimento do estudo, respeitou-se todas as diretrizes disciplinadas pela Resolução 466/2012 do Conselho Nacional da Saúde, sendo ele autorizado pela Secretaria Municipal de Saúde e aprovado pelo Comitê Permanente de Ética em Pesquisa com Seres Humanos da Universidade Estadual de Maringá (Parecer n 1.963.605/2017).

\section{Resultados}

Dentre os 35 jovens participantes, 21 (60,0\%) eram do sexo feminino, 34 (97,1\%) eram solteiros, 25 $(71,4)$ se autodeclaram de cor branca, $24(68,6 \%)$ tinham mais de 20 anos, 30 (85,7\%) residiam com os pais, 25 (71,4\%) estavam inseridos no mercado de trabalho, 19 (54,3\%) não possuíam plano de saúde, nove $(25,7 \%)$ tinham ensino superior completo e 11 $(31,4 \%)$ estavam na faculdade.

No que se refere à doença, 19 jovens $(54,3 \%)$ referiram que os sintomas iniciais surgiram ainda na infância e foram: perda de peso (12 - 63,1\%), poliúria $(10-52,3 \%)$ e polidipsia ( 8 - 42,1\%). Uma parcela significativa, 17 jovens (48,5\%), já precisou de internação devido à complicação da DM, sendo estas ocasionadas por hiperglicemia (11 - 64,7\%), hipoglicemia (5 - 29,4\%) e cetoacidose (1 - 5,8\%). Quanto ao controle da doença, todos possuíam glicosímetro, 30 
$(85,7 \%)$ verificavam a glicemia capilar diariamente, $31(88,6 \%)$ faziam uso de mais de um tipo de insulina e $32(91,4 \%)$ realizavam rodízio nos locais de aplicação. Ressalta-se que dois jovens (5,7\%) informaram utilizar bomba de insulina.

A autoeficácia psicossocial dos participantes em relação ao autogerenciamento do autocuidado em
DM foi considerada satisfatória, visto que 30 (85,7\%) tiveram a autoeficácia classificada como alta e cinco $(14,3 \%)$ como moderada (Tabela 1$)$. Na Tabela 2 , observa-se a caracterização sociodemográfica, perfil de saúde e busca por atendimento de saúde de acordo com a autoeficácia psicossocial dos jovens.

Tabela 1 - Distribuição das respostas de jovens com DM1 à Escala de Autoeficácia em Diabetes - Versão Curta

\begin{tabular}{|c|c|c|c|}
\hline Escala de Autoeficácia em Diabetes - Versão Curta & Não tem opinião & Está de acordo & $\begin{array}{c}\text { Está muito de } \\
\text { acordo }\end{array}$ \\
\hline Você sabe que coisas tem de fazer para cuidar da sua saúde, mas não gosta de fazer & - & 26 & 9 \\
\hline Você pode programar o seu dia a dia com coisas que vão te ajudar a cuidar da sua saúde & 8 & 20 & 7 \\
\hline $\begin{array}{l}\text { Você pode tentar coisas diferentes para afastar as dificuldades e fazer o que disse que ia } \\
\text { fazer para controlar a diabetes }\end{array}$ & 6 & 22 & 7 \\
\hline Você acredita que tem como achar coisas diferentes para fazer e sentir bem & 6 & 29 & - \\
\hline Você pode viver bem e dar um jeito de ir levando esse estresse todo da diabetes & 9 & 26 & - \\
\hline Quando precisar tem como você pedir ajuda para cuidar da diabetes & - & 30 & 5 \\
\hline Você sabe o que te faz ficar motivado para cuidar da diabetes & - & 31 & 4 \\
\hline $\begin{array}{l}\text { Você sabe bem como vocêé, não sabe? Então, dá para escolher direitinho o que vai dar certo } \\
\text { para você cuidar da sua saúde }\end{array}$ & - & 29 & 6 \\
\hline
\end{tabular}

Tabela 2 - Distribuição das variáveis perfil de saúde e busca por atendimento segundo a autoeficácia psicossocial de jovens com DM1

\begin{tabular}{|c|c|c|c|c|}
\hline \multirow[b]{2}{*}{ Variáveis } & \multicolumn{4}{|c|}{ Autoeficácia Psicossocial } \\
\hline & $\begin{array}{l}\text { Total } \\
\text { n (\%) }\end{array}$ & $\begin{array}{c}\text { Moderada } \\
\text { n (\%) }\end{array}$ & $\begin{array}{c}\text { Alta } \\
\text { n (\%) }\end{array}$ & p \\
\hline \multicolumn{5}{|l|}{ Perfil de saúde } \\
\hline \multicolumn{5}{|l|}{ Estado nutricional } \\
\hline Adequado & $19(54,3)$ & $4(21,1)$ & $15(78,9)$ & \multirow[t]{2}{*}{$0,466^{*}$} \\
\hline Excesso de peso & $16(45,7)$ & $1(6,2)$ & $15(93,8)$ & \\
\hline \multicolumn{5}{|c|}{ Prática de atividade física } \\
\hline Não & $20(57,1)$ & $3(15,5)$ & $17(85,0)$ & \multirow[t]{2}{*}{$0,727 *$} \\
\hline Sim & $15(42,9)$ & $2(13,3)$ & $13(86,7)$ & \\
\hline \multicolumn{5}{|c|}{ Acompanhamento com endocrinologista (meses) } \\
\hline$<6$ & $30(85,7)$ & $5(16,7)$ & $25(83,3)$ & \multirow[t]{2}{*}{$0,439^{\dagger}$} \\
\hline$>6$ & $5(14,3)$ & - & $5(100)$ & \\
\hline \multicolumn{5}{|c|}{ Acompanhamento com nutricionista (meses) } \\
\hline$<6$ & $18(51,4)$ & $3(16,7)$ & $15(83,3)$ & \multirow[t]{2}{*}{$0,528^{\dagger}$} \\
\hline$>6$ & $17(48,6)$ & $2(11,8)$ & $15(88,2)$ & \\
\hline \multicolumn{5}{|c|}{ Busca por atendimento } \\
\hline \multicolumn{5}{|c|}{ Acompanhamento na Unidade Básica de Saúde } \\
\hline Não & $9(25,7)$ & - & $9(100,0)$ & \multirow[t]{2}{*}{$0,385^{*}$} \\
\hline Sim & $26(74,3)$ & $5(19,2)$ & $21(80,8)$ & \\
\hline \multicolumn{5}{|c|}{ Acompanhamento no Plano de saúde/consultório } \\
\hline Não & $19(54,3)$ & $2(10,5)$ & $17(89,5)$ & \multirow[t]{2}{*}{$0,835^{*}$} \\
\hline Sim & $16(45,7)$ & $3(18,8)$ & $13(81,2)$ & \\
\hline \multicolumn{5}{|c|}{ Procura Unidade de Pronto Atendimento } \\
\hline Não & $27(77,1)$ & $5(18,5)$ & $22(81,5)$ & \multirow[t]{2}{*}{$0,459 *$} \\
\hline Sim & $8(22,9)$ & - & $8(100,0)$ & \\
\hline \multicolumn{5}{|l|}{ Procura Hospital } \\
\hline Não & $22(62,9)$ & $4(18,2)$ & $18(81,8)$ & \multirow[t]{2}{*}{$0,721^{*}$} \\
\hline Sim & $13(37,1)$ & $1(7,7)$ & $12(92,3)$ & \\
\hline
\end{tabular}




\section{Discussão}

Considerou-se como limitação deste estudo o pequeno número de participantes, o que impossibilita a realização de generalizações sobre os resultados obtidos, e ainda, a possibilidade dos resultados terem sido influenciados pelas características dos participantes - boa escolaridade associada ao fato de serem jovens - o que lhes permitia identificar com maior facilidade e rapidez as respostas que consideravam mais adequadas ao invés de representantes de sua realidade. De qualquer modo, seus resultados são relevantes para a prática clínica ao demonstrar e reforçar que os índices de autoeficácia podem direcionar o planejamento da assistência, especialmente aos jovens.

Índices mais elevados de autoeficácia foram observados nos jovens com idade entre 22 e 24 anos, com ensino superior (completo ou incompleto) e com maior tempo de diagnóstico, resultados estes que reforçam que a autoeficácia tende a evoluir à medida que as pessoas adquirem novas habilidades, experiências e conhecimentos ${ }^{(9)}$. Esse resultado corrobora com estudo realizado com 60 pessoas que tinham DM2 e idade entre 20 a 59 anos, no qual foi identificado que as pessoas com maior escolaridade apresentavam adesão adequada ao uso de medicamentos $(94,4 \%)$ e no seguimento de dieta saudável $(72,2 \%)^{(10)}$.

Os resultados alcançados, a autonomia para o autocuidado e a autoeficácia do indivíduo - o quanto ele acredita em si mesmo e em sua capacidade - são favorecidos por maior nível de escolaridade, por possibilitar sua compreensão quanto a importância do tratamento e de sua responsabilidade no modo e qualidade como ele é executado. Ressalta-se que em pessoas com diabetes, alto nível de autoeficácia, além de melhor controle da doença, também pode resultar em melhoria na saúde geral, saúde mental e funcionamento social, pois ela influencia a capacidade de autocuidado destas ${ }^{(9)}$. Este fato foi observado no presente estudo, pois das cinco pessoas com autoeficácia moderada, quatro já foram hospitalizadas duas ou mais vezes, por descontrole da doença.

Cabe ressaltar que, maiores níveis de escolaridade também favorecem a obtenção de recursos financeiros e, consequentemente, o cuidado e a manutenção do tratamento, pois insumos e produtos específicos para o controle da doença, geralmente são mais caros que os tradicionais $^{(2)}$. Estudo realizado com 16 pacientes, com idade entre 20 a 75 anos e diagnóstico de DM2, identificou que eles apresentavam dificuldades em nutrir-se de maneira saudável, cumprir o plano alimentar e associavam este fato a condições financeiras e ao alto custo de produtos indicados para pacientes com diabetes ${ }^{(11)}$.

Outro fator importante para o controle e prevenção de agravos é o diagnóstico precoce, pois possibilita o início imediato de medidas de controle e evita episódios de complicações agudas e crônicas. Neste sentido, para favorecer a adesão e o engajamento, é importante que os planos de cuidados, sempre que possível, levem em consideração as preferências dos usuários ${ }^{(2)}$.

Neste estudo, constatou-se que grande parte dos diagnósticos de DM1 ocorreu na infância, o que se assemelha a resultado de estudo realizado com 122 adolescentes (entre 12 e 18 anos), os quais relataram que a descoberta da doença ocorreu por volta dos nove anos, sendo que $60,0 \%$ deles foram diagnosticados até essa idade ${ }^{(12)}$.

Observou-se autoeficácia satisfatória em todos os jovens da pesquisa, independente do local onde eles buscam atendimento, o que reitera a importância do desenvolvimento de ações de promoção do autocuidado por parte dos profissionais de saúde e, de forma especial, pelos que integram as equipes da Estratégia Saúde da Família $^{(13)}$. Isto porque, ao trabalharem com uma população delimitada, possuem maior possibilidade de aproximar as pessoas da reflexão acerca de sua responsabilidade diante de sua própria saúde. No caso de jovens, a autonomia para o autocuidado é mais facilmente alcançada quando existe apoio da equipe multiprofissional de saúde, especialmente quando esta realiza acompanhamento contínuo, e com orientações e estímulos sobre o tratamento e cuidados específicos $^{(14)}$.

Ressalta-se que a Atenção Primária à Saúde dispõe de recursos necessários para diagnosticar, intervir e assistir os usuários com DM de maneira efetiva, promovendo a qualidade de vida e a redução de lesões nos órgãos-al- 
$\mathrm{vo}^{(13)}$. Isto é importante e precisa ser valorizado, pois a proporção da população que depende exclusivamente do atendimento dos serviços públicos é cada vez maior.

No que se refere à ocorrência de internações, identificou-se que a maioria dos participantes nunca necessitou de hospitalização em decorrência da DM1, o que se assemelha ao resultado de estudo realizado no Ceará, com 160 adolescentes (idade entre 10 e 19 anos) com DM1, o qual constatou que $71,3 \%$ deles nunca haviam sido internado por complicações da doença ${ }^{(15)}$. Destarte, conforme aponta estudo realizado na Austrália, estratégias de intervenção que enfoquem a educação em cuidados básicos à indivíduos com DM oferecem benefícios clinicamente importantes, pois reduzem a chance de complicações ${ }^{(16)}$. Nesse sentido, quanto mais o indivíduo investir em ações de autocuidado, menores as chances de complicações em especial as agudas, e de hospitalizações.

Outro fator que pode influenciar a baixa incidência de internação e de complicações é o suporte para o tratamento, identificado a partir da informação de que a maioria dos participantes residia com a família. As práticas de autocuidado são transferidas gradativamente dos pais para os filhos. Porém, no caso de doenças crônicas como DM, funções de acompanhamento, como vigiar e estimular o tratamento continuam sendo desenvolvidas por seus pais, o que tende a favorecer o controle glicêmico ${ }^{(3)}$. Além disso, outras variáveis modificáveis podem interferir neste controle, tais como qualidade das relações familiares, alfabetização em saúde e interações do jovem com o sistema de saúde ${ }^{(17)}$.

No caso de jovens, a participação familiar não substitui o acompanhamento profissional multidisciplinar, o qual proporciona uma assistência completa, pessoalizada e especializada, levando-se em conta fatores como dosagem hormonal, faixa etária, hábitos alimentares e culturais e necessidades cognitivas e psicossociais. Desta forma, a recomendação de consultas regulares com endocrinologista e nutricionista deve sempre ser estimulada entre os jovens com DM1 ${ }^{(1)}$.

Destarte, atenção diferenciada deve ser dispensada ao público jovem. Estudo de corte realizado na Austrália avaliou o impacto da participação/acompanhamento em serviços específico para jovens nas internações registradas no período de um ano em todos os hospitais da região e identificou a ocorrência de 55 internações, referentes a 39 pacientes jovens com DM1 - idade entre 15 e 25 anos. Constatou que a maioria das admissões $(82,0 \%)$ foram de jovens não apoiados por serviço específicos para eles e que o tempo de internação também foi significativamente maior neste grupo $^{(18)}$.

É importante ressaltar que adotar comportamentos de autocuidado exige do indivíduo antes de tudo que ele acredite que a doença lhe é prejudicial e que pode lhe causar danos graves, que os comportamentos a serem adotados são efetivos no enfrentamento e controle da doença e que as dificuldades na implementação de ações e comportamentos específicos são superadas por seus benefícios. Alguns comportamentos, no entanto, exigem habilidades técnicas e cognitivas e estas normalmente estão associadas às crenças de autoeficácia. Deste modo, como a autoeficácia influencia a pessoa a adotar ou não determinado comportamento e a superar os obstáculos que possam surgir ${ }^{(19)}$, ela deve ser estimulada pelos profissionais de saúde, mediante, por exemplo, entrevista motivacional que levará o jovem a refletir sobre suas ações de autocuidado.

Programa intensivo e prático de educação em diabetes, que incluiu parte teórica de conhecimento sobre a doença, dieta, exercício, insulina e hipoglicemia, autoanálise, autogestão, complicações macro e microvasculares e uma parte prática, que envolveu treinamento de habilidades (automonitoramento e autocuidado, injeção de insulina, contagem de carboidratos) e exercícios práticos (prevenir a hipoglicemia e agir quando ocorre, ajustar a dose de insulina, planejar o exercício), constatou que ele foi bastante eficaz em seis meses e um ano após a intervenção. Contudo, os autores asseveraram que para obter benefícios mais duradouros, no planejamento de programas de educação de pacientes com diabetes, recomenda-se incluir também estratégias psicológicas, de modo a motivá-los a fazer mudanças reais em seu estilo de vida $^{(20)}$.

Por fim, é importante destacar que o compor- 
tamento em saúde de indivíduos com doenças crônicas como o Diabetes Mellitus precisa ser avaliado com cautela e levar em consideração diversos fatores, inclusive a veracidade do autorrelato. De qualquer modo, conhecer as características deste comportamento pode subsidiar os profissionais de saúde na implementação de estratégias mais eficazes na obtenção de resultados positivos em relação ao controle da doença, a fim de favorecer a mensuração do impacto das intervenções implementadas e fortalecer a capacidade de enfrentamento da doença e seu tratamento.

\section{Conclusão}

Os resultados apontam que os jovens em estudo apresentavam autoeficácia satisfatória (moderada e alta), sem associação significativa com características sociodemográficas, perfil de saúde e busca por atendimento em saúde. Este resultado pode estar relacionado às características da população em estudo - jovens e com elevada escolaridade - o que favorece a adoção de comportamentos positivos em saúde.

\section{Colaborações}

Nass EMA, Marcon SS e Teston EF contribuíram com a concepção do projeto, análise e interpretação dos dados, redação do artigo, revisão crítica relevante do conteúdo intelectual e aprovação final da versão a ser publicada. Haddad MCFL, Reis P e Lino IGT contribuíram com a redação do artigo e aprovação final da versão a ser publicada.

\section{Referências}

1. American Diabetes Association. Summary of reviews: Healthcare Standards in Diabetes. Diabetes Care [Internet]. 2019 [cited ago 22, 2019]; 39(Suppl 1):45. Available from: https://care.diabetesjournals. org/content/42/Supplement_1

2. Bertin RL, Elizio NPS, Moraes RNT, Medeiros CO, Fiori LS, Ulbbrich AZ. Percepções do cotidiano alimentar de crianças e adolescentes com diabetes mellitus tipo 1 . Rev Contexto Saúde. 2016; 16(30):100-9. doi: https://doi. org/10.21527/2176-7114.2016.30.100-109

3. Ferreira LE, Zanatta EA, Brum MLB, Nothaft SC, Motta MGCM. Diabetes Mellitus sob a ótica do adolescente. Cogitare Enferm. 2013; 18(1):71-7. doi: http://dx.doi.org/10.5380/ce.v18i1.27045

4. Szwarcwald CL, Souza PRBJ, DamacenaGN, Almeida WS, Malta DC, Stopa SR, et al. Recommendations and practice of healthy behaviors among patients with diagnosis and diabetes in Brazil: National Health Survey (PNS), 2013. Rev Bras Epidemiol. 2015; 18(Suppl 2):132-45. doi: http://dx.doi. org/10.1590/1980-5497201500060012

5. Serrabulho L, Matos MG, Nabais J, Raposo J. Perspectivas de jovens adultos com diabetes tipo 1 sobre a sua vida e a diabetes. Rev Port Diabetes [Internet]. 2015 [citado 2019 mai 15]; 10(1):1528. Disponível em: https://dspace.uevora.pt/ rdpc/bitstream/10174/17463/1/ARTIGO\%20 QUALITATIVO\%20REVISTA\%20SPD\%202015. pdf

6. Ministério da Saúde (BR). E-SUS - Atenção Básica [Internet]. 2017 [citado 2019 ago. 05]. Disponível em:http://datasus.saude.gov.br/projetos/50-esus

7. Chaves FF, Reis IA, Pagano AS, Torres HC. Translation, cross-cultural adaptation and validation of the Diabetes Empowerment Scale - Short Form. Rev Saúde Pública. 2017; 51:16. doi: http://dx. doi.org/10.1590/s1518-8787.2017051006336

8. Onis M, Onyango AW, Borghi E, Siyam A, Nishida C, Siekmann J. Development of a WHO growth reference for school-aged children and adolescents. Bull World Heal Organ [Internet]. 2007 [cited ago 05, 2019]; 85(10):812- 819. Available from: https://www.who. int/bulletin/volumes/85/9/07-043497.pdf

9. Morrison G, Weston P. Self-efficacy: a tool for people with diabetes managed by continuous subcutaneous insulin in fusion.] Diabetes Nurs [Internet]. 2013 [cited jan 17, 2019]; 17:1. Available from: https://pdfs. semanticscholar.org/2762/8c816640fff39f9ce 8aa38c3ec284f528c58.pdf

10. Costa LJS, Machado ALG, Silva AZ, Braga CR, Costa JN, Formiga LMF. Self-care of young adults with type 2 diabetes mellitus. Rev Enferm UFPE on line [Internet]. 2016 [cited Jan 17, 2019]; 10(11):3875-82. 
Available from: https://periodicos.ufpe.br/revistas/ revistaenfermagem/article/view/11468

11. Cecílio SG, Brasil CLGB, Vilaça CP, Silva SMF, Vargas EC, Torres HC. Psychosocial aspects of living with diabetes mellitus in promoting self-care. Rev Rene. 2016; 17(1):44-51. doi: http://dx.doi.org/10.15253/21756783.2016000100007

12. Greco-Soares JP, Dell'Aglio DD. Adesão ao tratamento em adolescentes com diabetes mellitus tipo 1. Psicol Saúde Doenç. 2017; 18(2):322-34. doi: http://dx.doi. org/10.15309/17psd180204

13. Nascimento TMRV, Resnicow K, Nery M, Brentani A, Kaselitz E, Agrawal P, et al. A pilotstudy of a community health agent-led type 2 diabetes self-management program using Motivational Interviewing-based approaches in a public primary care center in São Paulo, Brazil. BMC Health Serv Res. 2017; 17:32. doi: http://dx.doi.org/10.1186/s12913-016-1968-3

14. Silva ANS, Serafim ARMR, Alcântara CM, Queiroz MVO. Experiências de adolescentes com diabetes tipo 1 e intervenções educativas multiprofissionais para o cuidado. Cienc Cuid Saude 2018; 17(2). doi: http:// dx.doi.org/10.4025/cienccuidsaude.v17i2.40434

15. Lopes JSO, Dutra LRS, Lima NMM, Moura DJM. Perfil Socioeconômico e clínico de adolescentes portadores de diabetes mellitus tipo 1 atendidos em um centro de referência de Fortaleza-CE. Rev Diál Acad [Internet]. 2015 [citado 2019 mar 9];4(2):136-44. Disponível em: http://revista.fametro.com.br/index.php/RDA/ article/download/99/104
16. Speight J, Holmes-Truscott E, Harvey DM, Hendrieckx C,HaggerVL, Harris SE, et al.Structured type 1 diabetes education delivered in routine care in Australia reduces diabetes-related emergencies and severe diabetes-related distress: The Ozdafne program. Diabetes Res Clin Pract. 2016; 112:65-72. doi: http:// dx.doi.org/10.1016/j.diabres.2015.11.002

17. Agarwala S, Jawadb AF, Miller VA. A multivariate model exploring the predictive value of demographic, adolescent, and family factors on glycemic control in adolescents with type 1 diabetes. Pediatr Diabetes. 2016; 17(7):500-8. doi: http://dx.doi. org/10.1111/pedi.12331

18. Burns K, Farrell k, Myszka R, Park k, HolmesWalker DJ. Access to ayouth-specific service foryoung adults with type 1 diabetes mellitus is associated with decreased hospital length of stay for diabetic ketoacidosis. Internal Med J. 2017; 48(4):396-402. doi: https://doi.org/10.1111/imj.13649

19. Riegel B, Dickson VV, Garcia LE, Creber RM, Streur M. Mechanisms of change in self-care in adults with heart failure receiving a tailored, motivational interviewing intervention. Patient Educ Couns. 2017; 100(2):283-8. doi: http://dx.doi.org/10.1016/j. pec.2016.08.030

20. Ruiz-González I, Fernández-Alcántara FA, GuardiaArchilla T, Rodríguez-Morales S, Molina A, Casares $D$, et al. Long-term effects of an intensive-practical diabetes education program on HbA1c and selfcare. Appl Nurs Res. 2016; 31:13-8. doi: https:// doi.org/10.1016/j.apnr.2015.12.008 